\title{
Investigating the use of record-to-record variability in static capacity approaches
}

\author{
Pierre Gehl ${ }^{1}$, John Douglas ${ }^{1}$, Tiziana Rossetto ${ }^{2}$, Joshua Macabuag $^{2}$, \\ Arash Nassirpour ${ }^{2}$, Stylianos Minas $^{2}$ and Philippe Duffour ${ }^{2}$
}

${ }^{1}$ Risks and Prevention Division, BRGM, 3 avenue Claude Guillemin, 45060 Orléans, France; PH (33) 2.38.64.48.77; FAX (33) 2.38.64.47.38; email: p.gehl@brgm.fr

${ }^{2}$ EPICentre, Department of Civil, Environmental and Geomatic Engineering, University College London, Gower Street, London, United Kingdom

\begin{abstract}
In performance-based earthquake engineering, fragility curves constitute an essential component, providing a probabilistic link between the earthquake intensity measure and the structural response, expressed as an engineering demand parameter. In this study we apply a capacity spectrum method that uses inelastic response spectra derived from earthquake accelerograms to construct fragility curves for an example reinforced concrete structure. This approach has the advantage of simplicity and rapidity over methods using the accelerograms directly. Various sets of scaled and unscaled accelerograms are used to investigate the impact of record-to-record variability on the derived curves. It is found that the variability in the input spectra at periods longer than the natural period of the undamaged structure has a direct impact on the standard deviation associated with the curves. When the spectra closely match a target the curves are almost vertical (small standard deviations) but as the variation around the target increases the curves become flatter (high standard deviations). A variant of the peak-to-trough measure introduced by Stafford and Bommer (2010) provides a useful way of measuring the variability in the input spectra.
\end{abstract}

\section{INTRODUCTION}

In the context of earthquake engineering, fragility curves express the probability of a structure to experience a certain damage level given shaking of a particular strength. Therefore, they link seismic hazard and vulnerability and, consequently, they play a key role in performance-based earthquake engineering. Because of this important task much research has been conducted over the past couple of decades on the development of such functions and many dozens of curves have been published for various types of structures. As discussed by, for example, Calvi et al. (2006) such vulnerability models fall into three board categories: empirical (based on observed earthquake damage), analytical (based on structural modeling) and hybrid (combining aspects of the other two approaches). This article concerns fragility curves derived by the analytical approach. 
Within the analytical approach there is a separation between curves derived by static approaches based on pushover analyses and those developed using full nonlinear time-history calculations. Here our focus is on the development of fragility curves derived using static calculations based on the capacity spectrum method (Freeman et al., 1975). Such an approach has the advantages of: rapidity, simplicity and, thanks to this simplicity, the ability to better understand the physical reasons for the results obtained. On the other hand, because they simplify dynamic structural response to a static problem the results may not be as accurate as those obtained by time-history analyses. A comparison between the results of the two approaches is made in the companion article by Rossetto et al. (2014).

The capacity spectrum approach used here characterizes earthquake shaking solely by a response spectrum based on a series of single-degree-of-freedom (SDOF) systems. Consequently much of the true variability in earthquake ground motions is lost. For example, response spectra are 'blind' to the duration of shaking and, therefore, two records, one of short duration and one much longer, but with the same spectrum would be assessed by this approach as having the same influence on the structure. In reality this is unlikely to be true. Nevertheless, response spectra of earthquake ground motions do show considerable variability, even for the same magnitude and distance, and these differences will influence the fragility curves derived using the capacity spectrum method. The purpose of this article is to understand the influence of this variability in response spectrum on the full fragility curves: how much of the 'true' variability in structural response is lost by using a classic static approach that does not account for spectral variability?

In the next section the approach followed here to assess the fragility of structures and the computer program written to easily implement this technique are described. The following section presents the example structure and its structural behavior as characterized by its pushover curve. Then the different accelerograms used as input to measure the fragility of this structure are presented. In the penultimate section the derived fragility curves are given and the impact of the spectral variability on these curves discussed. The article ends with some conclusions and suggestions for future work.

\section{CAPACITY SPECTRUM APPROACH TO DERIVE FRAGILITY CURVES}

The capacity spectrum approach, originally proposed by Freeman et al. (1975), relies on the determination of a structure's performance point by comparing the equivalent capacity and demand spectra in terms of acceleration-displacement response spectra (ADRS). Several approaches have then been proposed to match the target inelastic response spectra, such as the ATC-40 approach (ATC, 1996), the coefficient method in FEMA-356 (FEMA, 2000) and the N2 method by Fajfar $(1999,2000)$.

The approaches cited above usually require a normalized design spectra and the use of a corner period $\left(\mathrm{T}_{\mathrm{c}}\right)$ to identify acceleration- and displacement-sensitive segments of the spectra. Therefore, these standardized spectra are commonly defined as smooth 
functions that do not account for the variability present in natural spectra. Some recent studies (Faella et al., 2008) show that natural (unsmooth) response spectra can be used in the N2 method thereby accounting for record-to-record variability in the performance-point evaluation. In the present study the capacity spectrum method that has been originally proposed by Rossetto (2004) and Rossetto and Elnashai (2005) is investigated. This differs from those cited above by directly using acceleration timehistories from which both elastic and inelastic spectra are computed and used to find the performance point. This method has been recently translated into a Matlab-based automated tool (Rossetto et al., 2014) known as FRACAS ("FRAgility from Capacity Spectrum assessment"). The main steps of this procedure are the following (Figure 1).
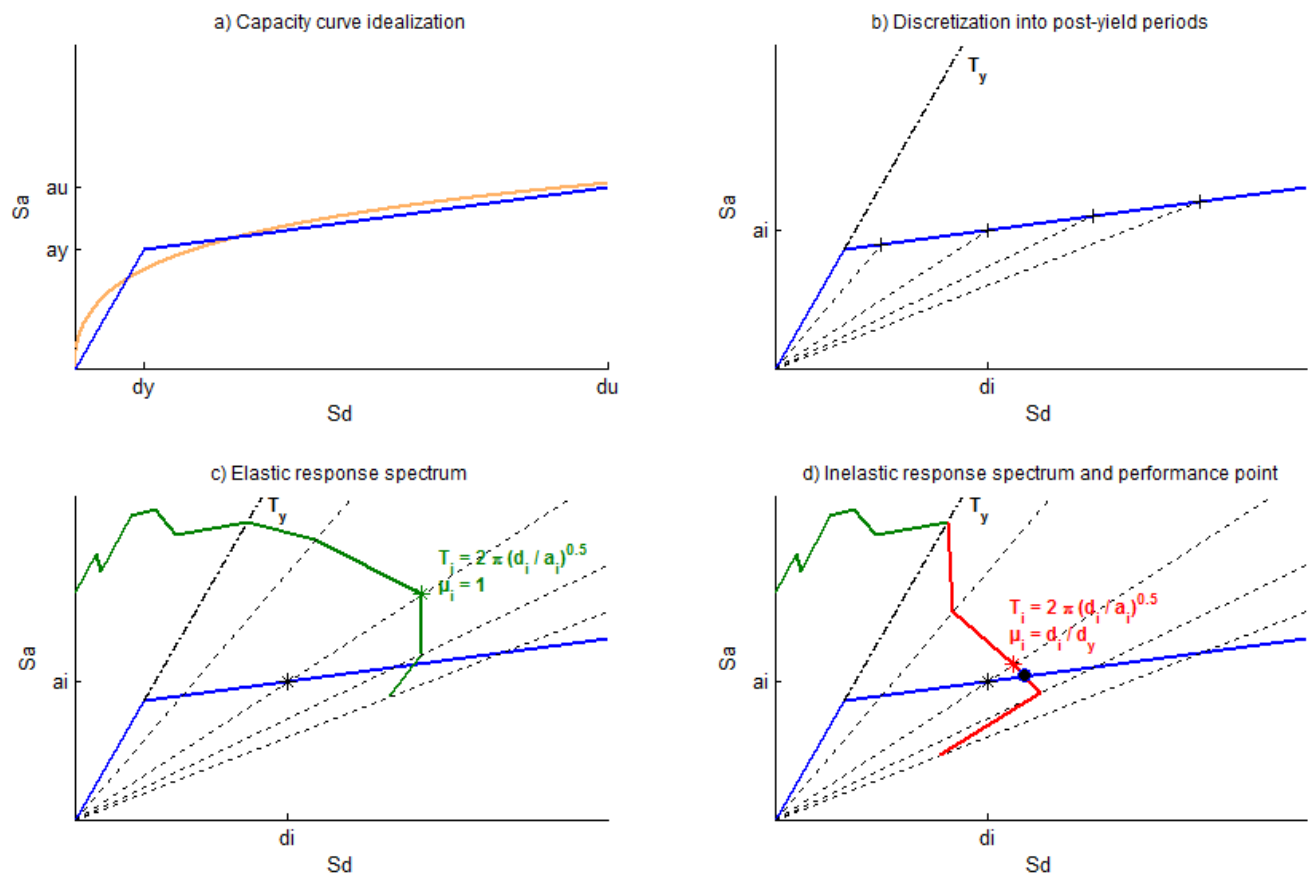

Figure 1 - Summary of the main steps carried out by FRACAS for a bilinear capacity curve

1. Transformation of the pushover curve into a capacity curve in ADRS space, through the use of relative floor displacements and floor masses.

2. Idealization of the capacity curve making various choices regarding the selection of the yield and ultimate points, the number of segments (bilinear or trilinear) and the presence of strain hardening. The idealized curve is discretized by selecting various periods as analysis points.

3. Input of the acceleration time-history and computation of the corresponding elastic response spectrum, until the yield period (elastic limit) is reached.

4. For each of the selected periods, computation of the inelastic response spectrum. For this, iterative Newmark integration is performed at each time step and a restoring force is used to account for the stiffness reduction in the inelastic branches of the model. An optimization process finds the closest equivalent SDOF model in terms of target ductility and effective period. 
5. Using both elastic and inelastic parts of the response spectrum, estimation of the performance point as the intersection of the capacity and response spectra. No iterative process is required at this stage because the generated inelastic spectrum already has the required ductility and stiffness.

6. Repetition of the analysis for a different pushover curve (if studying many structures) and a different accelerogram to generate many performance points.

7. Conversion of the performance points (expressed in ADRS format) into the desired intensity measures (IMs) and engineering demand parameters (EDPs), e.g. spectral acceleration (SA) and inter-story drift ratio (ISDR).

8. Derivation of the corresponding fragility curves for various damage states.

It is important to note that, in contrast to other capacity spectrum methods, FRACAS does not rely on reduction factors or indices to estimate the inelastic spectrum from the elastic one. Instead, it carries out, for each target ductility and period, a simplified dynamic analysis on the idealized nonlinear SDOF model corresponding to the capacity curve. This process proves to be more time-consuming than the commonlyused static approaches but it remains faster and more robust than performing full time-history analyses on finite element models. This feature also has the advantage of permitting the use of various natural accelerograms that generate unsmoothed spectra as opposed to standardized design spectra. Therefore, the record-to-record variability can be directly introduced and the resulting cloud of performance points leads to fragility curves that account for the natural variability in the seismic demand.

\section{CONSIDERED STRUCTURE AND PUSHOVER CURVE}

The FRACAS procedure is applied to a model of a structure tested during the ICONS experiments (Pinto et al., 2002) using pseudo-dynamic loading. It is a four-story bareframe reinforced-concrete structure, which is assumed to be representative of common Mediterranean buildings dating from the 1960s to 1980s (Carvalho et al., 1999). A finite element model was developed using the Seismostruct software and the resulting capacity curve, based on a triangular loading pattern, is represented in Figure 2.

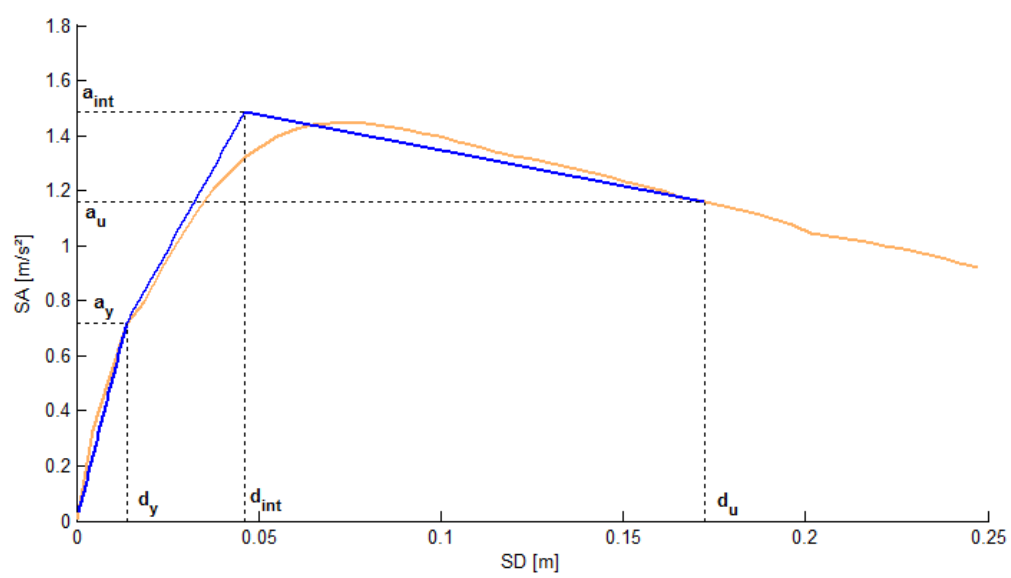

Figure 2 - Capacity curve of the structure (orange) and corresponding idealization (blue) performed by FRACAS. The yield $\left(d_{y}\right)$, ultimate $\left(d_{u}\right)$ and intermediate $\left(d_{i n t}\right)$ points of the idealization are shown. 
A trilinear idealization with stain hardening was chosen to account for the progressive degradation of the initial stiffness. The yield point (i.e. end of the elastic segment) is estimated by checking the region of the curve that experiences a relative change of gradient greater than $6 \%$, and an absolute gradient that is less than $50 \%$ of the initial one. This period differs from the one estimated by modal analysis (i.e. $0.68 \mathrm{~s}$ ): in the present study, SDOF equivalence is assumed, which may explain the differences in the elastic period because of different estimated yield points for the static analysis. The ultimate point (i.e. end of the curve) is then defined as the point at which the strength is $20 \%$ lower than the maximum. Finally, the intermediate point is obtained by minimizing the area between the real and idealized curves.

\section{DESIGN SPECTRUM AND INPUT ACCELEROGRAMS}

Because our aim is to understand the influence of response spectral variability on fragility curves we use accelerograms selected or generated based on the match of their spectra to a design spectrum. This approach is quite common in fragility analyses because of its link to seismic design code requirements, although true earthquake ground motions rarely match code spectrum and hence this approach is likely to underpredict the true variability in structural response. In spite of this potential limitation, here we use these sets of accelerograms proposed by Causse et al. (2013) for use on stiff soil (Site B) in combination with Eurocode 8 for medium hazard parts of France (design peak ground acceleration of $0.22 \mathrm{~g}$ ) (Figure 3):

1. Ten natural accelerograms with spectra closely matching the code spectrum over the period range 0.1 to 1s (SCALED - Eurocode 8);

2. The same accelerograms adjusted using wavelets (Hancock et al., 2006) so that their spectra better match the target (MATCHED);

3. Ten accelerograms generated using SIMQKE (Gasparini and Vanmarcke, 1976), which show very little dispersion in their spectra and match the target very closely (SIMQKE); and, finally

4. A set of natural accelerograms that have been randomly selected without any seismological or code constraints (SCALED - no constraints).

The accelerograms are, initially, linearly amplitude scaled so that the SAs at the natural period of the undamaged structure $(0.87 \mathrm{~s})$ are the same for all records and, then, rescaled to different SAs to generate many IM-EDP pairs.

Stafford and Bommer (2010) postulate that, when deriving fragility curves accounting for ground-motion variability, the peak-to-trough variability in their response spectra should not be too small. They define the peak-to-trough variability by the standard deviation of the natural logarithm of spectral ordinates over a number of records and a range of response periods defined by the 'bandwidth', i.e. the range of periods surrounding a central period. This bandwidth roughly corresponds to the degree of structural nonlinearity that is expected and the contribution of higher mode effects. Because within the capacity spectrum method used here the structure is not affected by spectral ordinates with periods shorter than the natural period (here $0.87 \mathrm{~s}$ ), we 
modify the definition of the peak-to-trough variability to account for only those periods longer than the natural period. Consequently, the graph (Figure 4) showing this variability is plotted in terms of period rather than bandwidth. Using the ground motion prediction equations (GMPEs) of Boore and Atkinson (2008), evaluated for an $\mathrm{Mw} 6$ earthquake at $10 \mathrm{~km}$ at a stiff soil site, and the model of Baker and Jayaram (2008) predicting the period-to-period correlation of spectral ordinates, a thousand response spectra were generated using random numbers generated from a multivariate normal distribution (Baker and Cornell, 2006). The peak-to-trough variability of these spectra was computed in the same way as for the four sets of accelerograms and is also plotted on Figure 4.
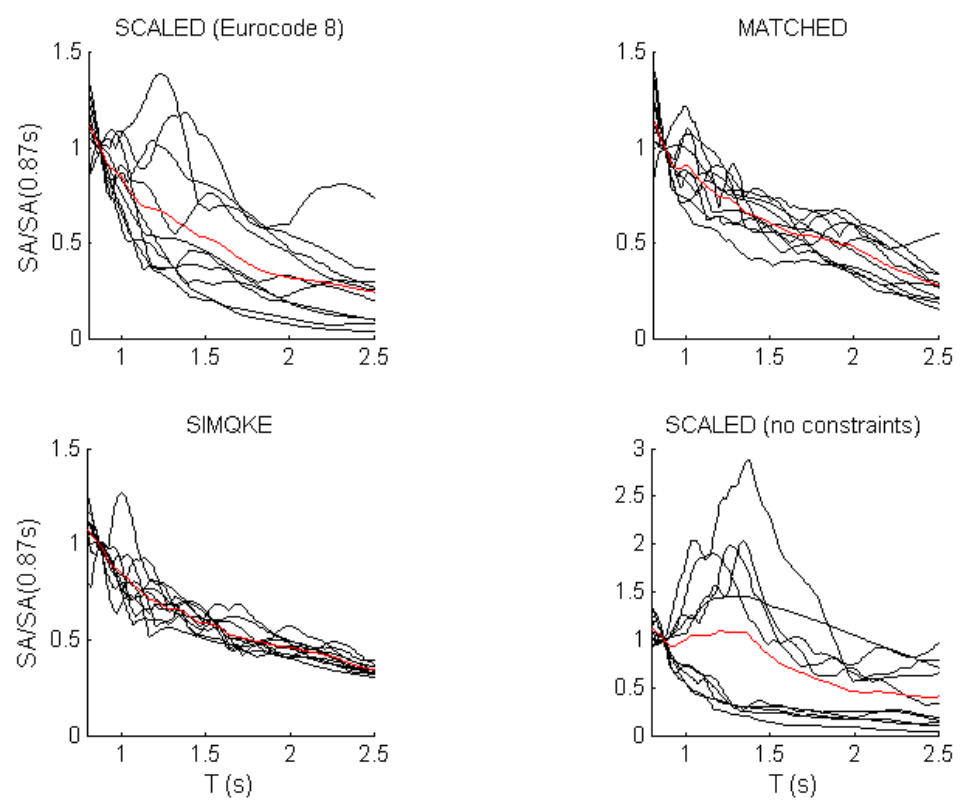

Figure 3 - Elastic response spectra of the four sets of accelerograms (in black) and corresponding average (in red). Only periods longer than the natural period are shown because this is the range of interest when applying the capacity spectrum method.

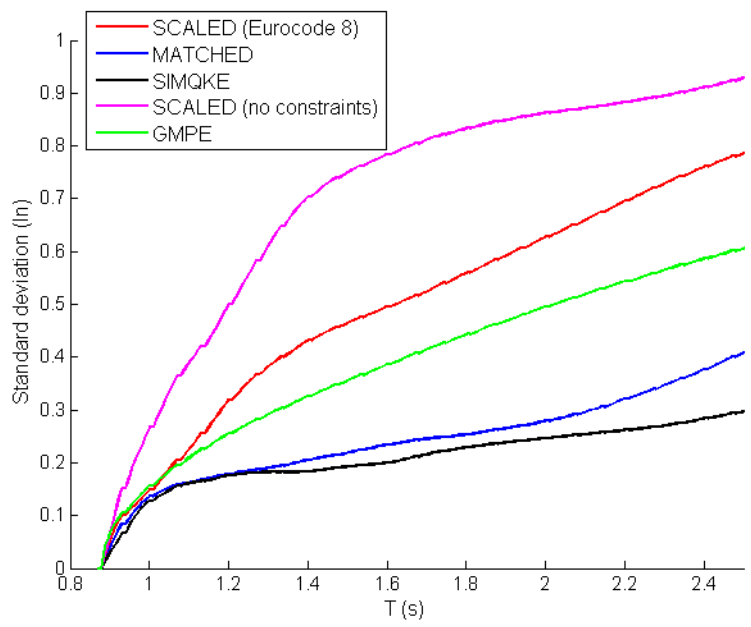

Figure 4 - Peak-to-through variability (using only periods longer than 0.87 s) of accelerograms 
The ultimate displacement of the structure is $d_{u}=0.1725 \mathrm{~m}$ (Figure 2) and, therefore, it is expected that its response is affected by periods up to $2 \pi \cdot\left(\mathrm{d}_{\mathrm{u}} / \mathrm{a}_{\mathrm{u}}\right)^{0.5}=2.4 \mathrm{~s}$. Figure 4 shows that, as expected, the SIMQKE records show considerably less spectral variability whereas the natural spectrally-matched records show slightly less dispersion than the natural accelerograms. The behavior predicted by the GMPEs and period-to-period correlation models falls between the variability from the scaled and matched accelerograms. The variability shows similar behavior to that shown by Stafford and Bommer (2010, their Figure 4.1) but higher absolute values.

FRACAS analysis of the test structure with each of the ground-motion sets, scaled to varying SAs, leads to a differing variability in the structural response (Figure 5). The use of scaled accelerograms allows the computation of the standard deviation of the maximum ISDR within each "stripe" of outputs (Figure 6). It can be noted that the variability in the structural response increases with the imposed intensity level: this observation emphasizes the role of the specific nonlinear computations that are performed in FRACAS during the estimation of the inelastic response spectrum. Once the yield limit is reached, the relation between the IM and the structural response shows high heteroscedasticity. The relative variability between the four sets of accelerograms also follows the same trend as the peak-to-trough variability. The dispersion in the structural response tends to stabilize or even decrease for higher intensities because performance points exceeding the ultimate point are considered as "collapse" events, and their value is just set at the last point of the curve.
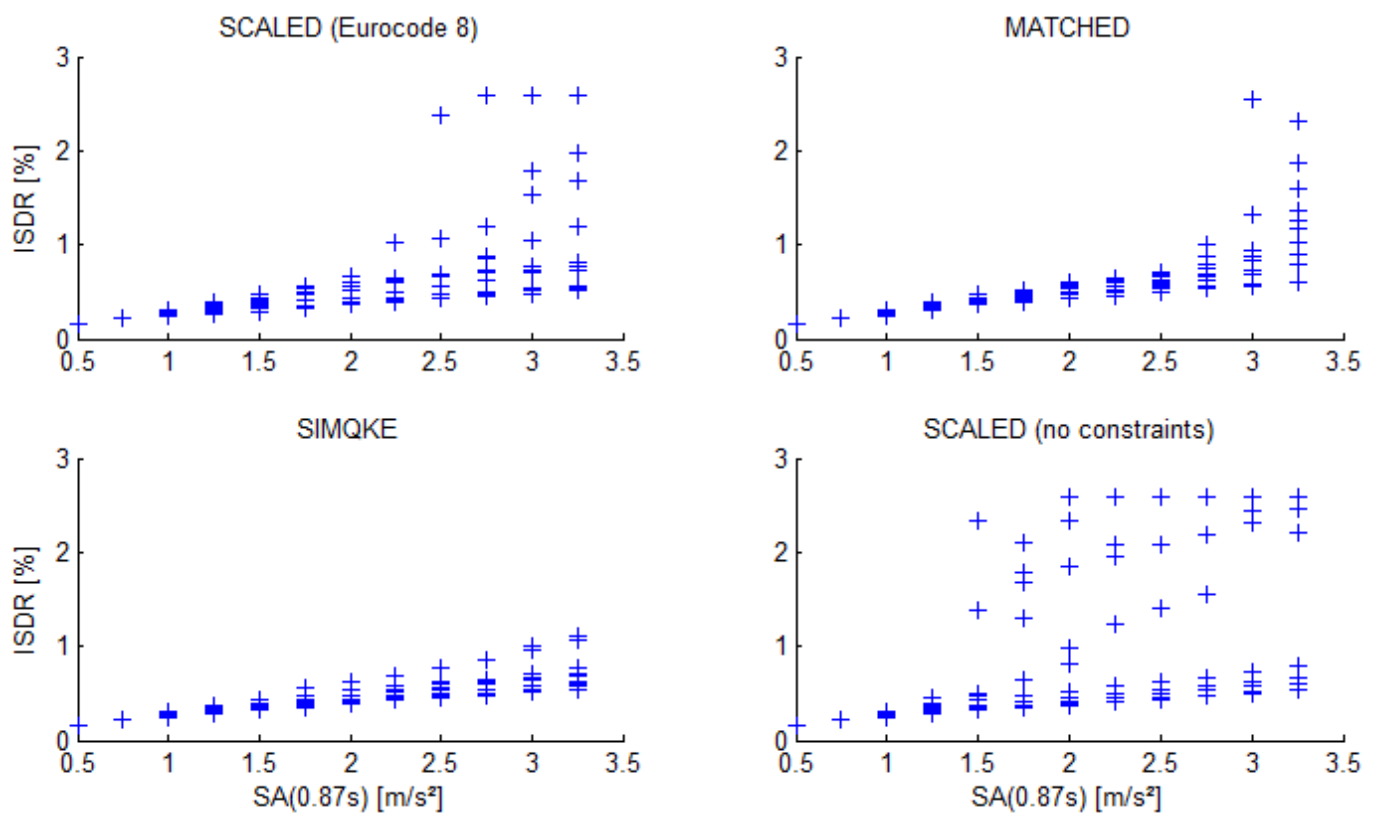

Figure 5 - Variability in the structural response (maximum ISDR) for the four sets of accelerograms 


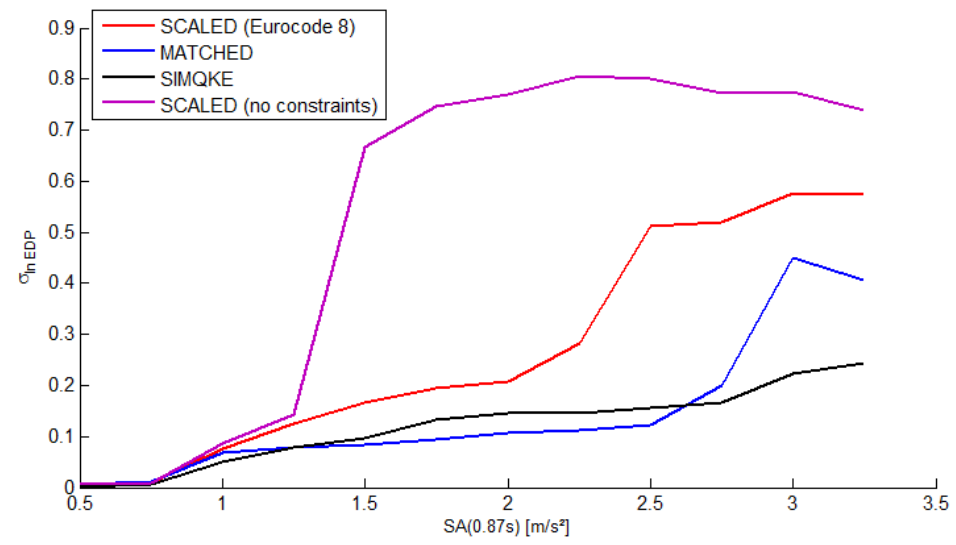

Figure 6 - Standard deviation of In ISDR as a function of spectral acceleration

\section{DERIVED FRAGILITY CURVES}

For the sake of the demonstration, a hypothetical performance target is defined for the building, chosen at the drift ratio threshold of $0.5 \%$. This value roughly corresponds to the usual limits for moderate or extensive damage (e.g. Ghobarah 2004; FEMA, 2000) and it has been chosen here, so that approximately half of the performance points are above and below this value. Fragility curves are derived for each set of accelerograms (i.e. 120 data points each) using a generalized linear model and probit as the link function (Baker, 2013). The resulting curves, therefore, follow a cumulative lognormal distribution, with the median $\alpha$ and the standard-deviation $\beta$ as the fragility parameters (Figure 7).

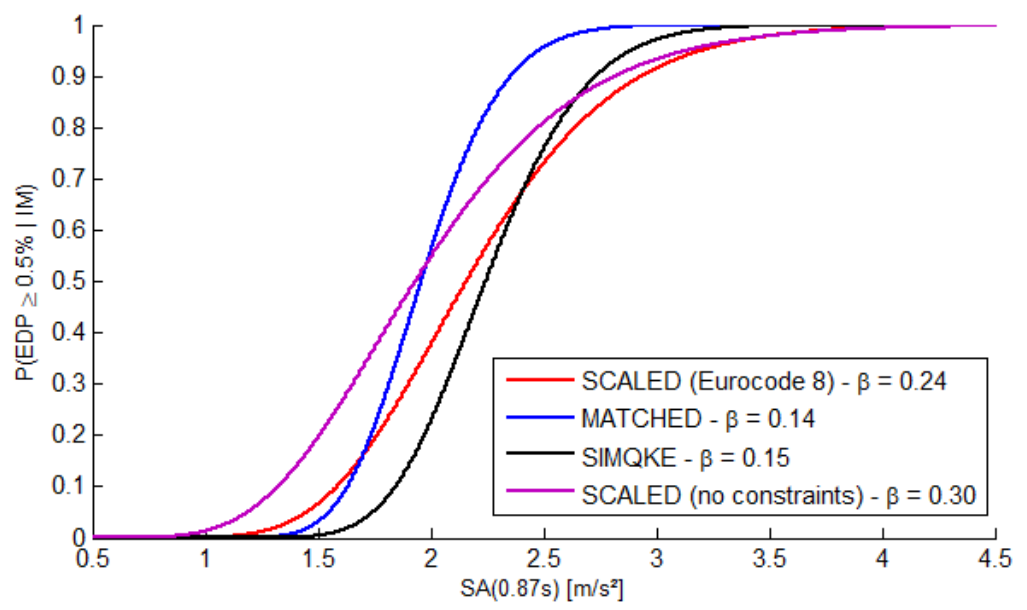

Figure 7.Fragility curves derived from the four sets of accelerograms for a given performance level $($ ISDR $=0.5 \%)$

It can be seen that the initial record-to-record variability is efficiently translated to the final fragility curves, as shown by the different values of the standard deviation $\beta$. The reason for the slightly different medians $(\alpha)$ could be related to the bias introduced by spectral matching recently evidenced by Seifried (2013). This observation raises the question whether the natural record-to-record variability, that is originally not present for matched records, could be added back in during the final steps. 


\section{CONCLUSIONS}

Using a capacity spectrum approach to derive fragility functions, we have demonstrated that the variability in spectral ordinates for periods beyond the natural period of the undamaged structure is directly correlated to the standard deviations of the curves. A variant of the peak-to-trough measure of the variability in the input spectra (accounting only for periods longer than the natural period) provides a useful measure of this variability. Consequently, differences between fragility curves derived using static pushover approaches can be partially explained by differences in the input spectra, even if the mean target spectra are similar. Correlations between the peak-to-trough variability and the standard deviation of the fragility curves $(\beta)$ could be investigated, although these correlations will be specific to the test structure.

\section{ACKNOWLEDGEMENTS}

Pierre Gehl's contribution to this study was partially funded by a Carnot Mobility grant. We also thank the MARS research project, coordinated by EDF, for its financial support.

\section{REFERENCES}

ATC (1996). "ATC-40- Seismic evaluation and retrofit of concrete buildings." Report, ATC-40, Applied Technology Council, Redwood City, CA.

Baker, J. W. (2013). "Trade-offs in ground motion selection techniques for collapse assessment of structures." Vienna Congress on Recent Advances in Earthquake Engineering and Structural Dynamics 2013, C. Adam, R. Heuer, W. Lenhardt \& C. Schranz (eds), Paper No. 123.

Baker, J.W. and Cornell, C.A. (2006). "Correlation of Response Spectral Values for Multi-Component Ground Motions." Bulletin of the Seismological Society of America, 96 (1), 215-227.

Baker, J.W. and Jayaram, N. (2008). "Correlation of spectral acceleration values from NGA ground motion models." Earthquake Spectra, 24 (1), 299-317.

Boore, D. M. and Atkinson, G. M. (2008). "Ground-motion prediction equations for the average horizontal component of PGA, PGV, and 5\%-damped PSA at spectral periods between $0.01 \mathrm{~s}$ and 10.0 s." Earthquake Spectra, 24(1), 99138.

Calvi, G. M., Pinho, R., Magenes, G., Bommer, J. J., Restrepo-Vélez, L. F. and Crowley, H. (2006). "Development of seismic vulnerability assessment methodologies over the past 30 years." ISET Journal of Earthquake Technology, 43(3), 75-104. Paper No. 472.

Carvalho, E.C., Coelho, E. and Campo-Costa, A. (1999). Preparation of the full-scale tests on reinforced concrete frames - Characteristics of the test specimens, materials and testing conditions. Report LNEC, Lisbon.

Causse, M., Laurendeau, A., Perrault, M., Douglas, J., Bonilla, L. F. and Guéguen, P. (2013). "Eurocode 8-compatible synthetic time-series as input to dynamic analysis." Bulletin of Earthquake Engineering, resubmitted after reviews. 
Fajfar, P. (1999). "Capacity spectrum method based on inelastic demand spectra". Earthquake Engineering and Structural Dynamics, 28, 979-993.

Fajfar, P. (2000). "A nonlinear analysis method for performance based seismic design.” Earthquake Spectra, 16(3), 573-592.

Faella, C., Lima, C. and Maritnelli, E. (2008). "Non-linear static methods for seismic fragility analysis and reliability evaluation of existing structures." Proceedings of the $14^{\text {th }}$ World Conference on Earthquake Engineering, Beijing, China.

FEMA (2000). "FEMA-356 - Prestandard and commentary for the seismic rehabilitation of buildings." FEMA, Publication $n^{\circ} 356$, The American Society of Civil Engineers for the Federal Emergency Management Agency, Washington, DC.

Freeman, S. A., Nicoletti, J. P. and Tyrell, J. V. (1975). "Evaluations of existing buildings for seismic risk - A case study of Puget Sound Naval Shipyard, Bremerton, Washington." Proceedings of the U.S. National Conference on Earthquake Engineering, 113-122.

Gasparini, D. A. and Vanmarcke, E. H. (1976). "SIMQKE: A Program for Artificial Motion Generation." Department of Civil Engineering, Massachusetts Institute of Technology, Cambridge, USA.

Ghobarah, A. (2004). "On drift limits with different damage levels." Proceeding of International Workshop on Performance-based seismic design concepts and implementation, Bled.

Hancock, J., Watson-Lamprey, J., Abrahamson, N., Bommer, J., Markatis, A., McCoy, E. and Mendis, R. (2006). "An improved method of matching response spectra of recorded earthquake ground motion using wavelets." Journal of Earthquake Engineering, 10(1), 67-89.

Pinto, A.V., Molina, J., Varum, H., Verzeletti, G., Coelho, E. and Carvalho, E.C. (2002). Pseudo-dynamic tests on a full-scale model of existing RC frames (bare frame and selective repair frame). Report EUR, EC, Joint Research Centre, Ispra, Italy.

Rossetto, T. (2004). Vulnerability curves for the seismic assessment of reinforced concrete building populations, $\mathrm{PhD}$ Thesis, Department of Civil \& Environmental Engineering, Imperial College, London.

Rossetto, T., and Elnashai, A. (2005). "A new analytical procedure for the derivation of displacement-based vulnerability curves for populations of RC structures." Engineering Structures, 27, 397-409.

Rossetto, T., Gehl, P., Minas, S., Nassirpour, A., Macabuag, J., Duffour, P. and Douglas, J. (2014). "Sensitivity analysis of different capacity approaches to assumptions in the modeling, capacity and demand representations." $A S C E$ ICVRAM-ISUMA conference, submission ID 319.

Seifried, A. E. (2013). Response spectrum compatibilization and its impact on structural response assessment, $\mathrm{PhD}$ thesis, Stanford University.

Stafford, P. J. and Bommer, J. J. (2010). "Theoretical consistency of common record selection strategies in performance-based earthquake engineering." Advances in Performance-Based Earthquake Engineering, Geotechnical, Geological and Earthquake Engineering, 13, 49-58. 